\title{
Finding and Visualizing Graph Clusters Using PageRank Optimization
}

\author{
Fan Chung Alexander Tsiatas \\ Department of Computer Science and Engineering \\ University of California, San Diego
}

\begin{abstract}
We give algorithms for finding graph clusters and drawing graphs, highlighting local community structure within the context of a larger network. For a given graph $G$, we use the personalized PageRank vectors to determine a set of clusters, by optimizing the jumping parameter $\alpha$ subject to several cluster variance measures in order to capture the graph structure according to PageRank. We then give a graph visualization algorithm for the clusters using PageRank-based coordinates. Several drawings of real-world data are given, illustrating the partition and local community structure.
\end{abstract}

\section{Introduction}

Finding smaller local communities within a larger graph is a well-studied problem with many applications. For example, advertisers can more effectively serve niche audiences if they can identify their target communities within the larger social web, and viruses on technological or population networks can be effectively quarantined by distributing antidote to local clusters around their origins [9].

There are numerous well-known algorithms for finding clusters within a graph, including $k$-means $[22,25]$, spectral clustering [29, 34], Markov cluster algorithms [13], and numerous others [19, 26, 27, 28, 30]. Many of these algorithms require embedding a graph into low-dimensional Euclidean space using pairwise distances, but graph distance-based metrics fail to capture graph structure in real-world networks with small-world phenomena since all pairs of nodes are connected within short distances. PageRank provides essential structural relationships between nodes and is particularly well suited for clustering analysis. Furthermore, PageRank vectors can be computed more efficiently than performing a dimension reduction for a large graph.

In this paper, we give clustering algorithms PageRank-Clustering that use PageRank vectors to draw attention to local graph structure within a larger network. PageRank was first introduced by Brin and Page [6] for Web search 
algorithms. Although the original definition is for the Web graph, PageRank is well defined for any graph. Here, we will use a modified version of PageRank, known as personalized PageRank [20], using a prescribed set of nodes as a seed vector.

PageRank can capture well the quantitative correlations between pairs or subsets of nodes, especially on small-world graphs where the usual graph distances are all quite small. We use PageRank vectors to define a notion of PageRank distance which provides a natural metric space appropriate for graphs.

A key diffusion parameter in deriving PageRank vectors is the jumping constant $\alpha$. In our clustering algorithms, we will use $\alpha$ to control the scale of the clustering. In particular, we introduce two variance measures which can be used to automatically find the optimized values for $\alpha$. We then use PageRank vectors determined by $\alpha$ to guide the selection of a set of centers of mass and use them to find the clusters via PageRank distances. We further apply our clustering algorithm to derive a visualization algorithm PageRank-Display to effectively display local structure when drawing large networks.

The paper is organized as follows: The basic definitions for PageRank are given in Section 2. In Section 3, we describe two cluster variance measures using PageRank vectors, and we give clustering algorithms in Section 4, with analysis in Section 5. A graph drawing algorithm is given in the last section and several examples are included.

\section{Preliminaries}

We consider general undirected graphs $G=(V, E)$ with vertex set $V$ and edge set $E$. For a vertex $v$, let $d_{v}$ denote the degree of $v$ which is the number of neighbors of $v$. For a set of nodes $T \subseteq V$, the volume of $T$ is defined to be $\operatorname{vol}(T)=$ $\sum_{v \in T} d_{v}$. Let $D$ denote the diagonal degree matrix and $A$ the adjacency matrix of $G$, where

$$
A_{i j}= \begin{cases}1 & \text { if }\left\{v_{i}, v_{j}\right\} \in E \\ 0 & \text { otherwise }\end{cases}
$$

We consider a typical random walk on $G$ with the transition probability matrix defined by $P=D^{-1} A$ and we denote the lazy walk by $W=(I+P) / 2$. Let $\pi=\vec{d} / \operatorname{vol}(G)$ denote the stationary distribution of the random walk, if it exists. Personalized PageRank vectors are based on random walks with two governing parameters: a seed vector $\vec{s}$, representing a probability distribution over $V$, and a jumping constant $\alpha$, controlling the rate of diffusion. The personalized PageRank vector $\operatorname{pr}(\alpha, \vec{s})$ is defined to be the solution to the following recurrence relation:

$$
\operatorname{pr}(\alpha, \vec{s})=\alpha \vec{s}+(1-\alpha) \operatorname{pr}(\alpha, \vec{s}) W .
$$

Here, $\vec{s}$ (and all other vectors) will be treated as row vectors. The original definition of PageRank defined in [6] is the special case where the seed vector 
is the the uniform distribution. If $\vec{s}$ is simply the distribution which is 1 for a single node $v$ and 0 elsewhere, we write $\operatorname{pr}(\alpha, v)$.

An alternative expression for the personalized PageRank $\operatorname{pr}(\alpha, \vec{s})$ is a geometric sum of random walks (see [4]):

$$
\operatorname{pr}(\alpha, \vec{s})=\alpha \sum_{t=0}^{\infty}(1-\alpha)^{t} \vec{s} W^{t} .
$$

In general, it can be computationally expensive to compute PageRank exactly; it requires using the entire graph structure which can be prohibitive on large networks. Instead, we use an approximate PageRank algorithm as given in $[4,10]$. This approximation algorithm is much more tractable on large networks, because it can be computed using only the local graph structure around the starting seed vector $\vec{s}$. Besides $\vec{s}$ and the jumping constant $\alpha$, the algorithm requires an approximation parameter $\epsilon$.

For a subset of nodes $H$ in a graph $G$, the Cheeger ratio $h(H)$ is a measure of the cut between $H$ and its complement $\bar{H}$ :

$$
h(H)=\frac{e(H, \bar{H})}{\min (\operatorname{vol}(H), \operatorname{vol}(\overline{(} H)))},
$$

where $e(H, \bar{H})$ denotes the number of edges $\{u, v\}$ with $u \in H$ and $v \in \bar{H}$.

For a set of points $S=\left\{s_{1}, \ldots, s_{n}\right\}$ in Euclidean space, the Voronoi diagram is a partition of the space into disjoint regions $R_{1}, \ldots, R_{n}$ such that each $R_{i}$ contains $s_{i}$ and the region of space containing the set of points that are closer to $s_{i}$ than any other $s_{j}$. Voronoi diagrams are well-studied in the field of computational geometry. Here we consider Voronoi diagrams on graphs using PageRank vectors as a notion of closeness.

For two vertices $u, v$, we define the PageRank distance with jumping constant $\alpha$ as:

$$
\operatorname{dist}_{\alpha}(u, v)=\left\|\operatorname{pr}(\alpha, u) D^{-1 / 2}-\operatorname{pr}(\alpha, v) D^{-1 / 2}\right\| .
$$

Throughout this paper, $\|\cdot\|$ denotes the $L_{2}$-norm.

We can further generalize this distance to two probability distributions $p$ and $q$ defined on the vertex set $V$ of $G$. Namely, the PageRank distance, with jumping constant $\alpha$, between $p$ and $q$ is defined by

$$
\operatorname{dist}_{\alpha}(p, q)=\sum_{u, v} p(u) q(v) \operatorname{dist}(u, v) .
$$

With this definition, for a subset $S$ of vertices, we can generalize the notion of a center of mass for $S$ to be a probability distribution $c$. For a given $\epsilon>0$, we say $c$ is an $\epsilon$-center or center of mass for $S$ if

$$
\sum_{v \in S} \operatorname{dist}_{\alpha}(c, v) \leq \epsilon
$$

Let $C$ denote a set of $k$ (potential) centers. The goal is for each center $c$ to be a representative center of mass for some cluster of vertices. We let $R_{c}$ denote 
the set of all vertices $x$ which are closest to $c$ in terms of PageRank, provided the jumping constant $\alpha$ is given:

$$
R_{c}=\left\{x \in V: \operatorname{dist}_{\alpha}(c, x) \leq \operatorname{dist}_{\alpha}\left(c^{\prime}, x\right) \text { for all } c^{\prime} \in C\right\} .
$$

\section{PageRank Variance and Cluster Variance Mea- sures}

For a vertex $v$ and a set of centers $C$, let $c_{v}$ denote the center that is closest to $v$, (i.e., $c_{v}$ is the center of mass $c \in C$ such that $v \in R_{c}$ ).

We follow the approach as in $k$-means by defining the following evaluative measure for a potential set of $k$ centers $C$, using PageRank instead of Euclidean distances.

$$
\mu(C)=\sum_{v \in V} d_{v}\left\|\operatorname{pr}(\alpha, v) D^{-1 / 2}-\operatorname{pr}\left(\alpha, c_{v}\right) D^{-1 / 2}\right\|^{2}=\sum_{v \in V} d_{v} \operatorname{dist}_{\alpha}\left(v, c_{v}\right)^{2} .
$$

Selecting a set of representative centers within a graph is a hard problem, known to be NP-complete. There are many approximate and heuristic algorithms used in practice (see [33]). Here, we will develop algorithms that use personalized PageRank vectors to select the centers. In the Web graph, links between websites can be interpreted as votes for a website's importance, and PageRank vectors are used to determine which pages are intrinsically more important in the overall graph. Personalized PageRank vectors are local information quantifying the importance of every node to the seed. Thus, the $u$ th component of the personalized PageRank vector $\operatorname{pr}(\alpha, v)$ quantifies how wellsuited $u$ is to be a representative cluster center for $v$.

To evaluate a set of cluster centers in a graph $G$, we consider two measures that capture the community structure of $G$ with respect to PageRank:

$$
\begin{aligned}
\Phi(\alpha) & =\sum_{v \in V} d_{v}\left\|\operatorname{pr}(\alpha, v) D^{-1 / 2}-\operatorname{pr}(\alpha, \operatorname{pr}(\alpha, v)) D^{-1 / 2}\right\|^{2} \\
& =\sum_{v \in V} d_{v} \operatorname{dist}_{\alpha}(v, \operatorname{pr}(\alpha, v))^{2} \\
\Psi(\alpha) & =\sum_{v \in V} d_{v}\left\|\operatorname{pr}(\alpha, \operatorname{pr}(\alpha, v)) D^{-1 / 2}-\pi D^{-1 / 2}\right\|^{2} \\
& =\sum_{v \in V} d_{v} \operatorname{dist}_{\alpha}(\operatorname{pr}(\alpha, v), \pi)^{2}
\end{aligned}
$$

The $\alpha$-PageRank-variance $\Phi(\alpha)$ measures discrepancies between the personalized PageRank vectors for nodes $v$ and possible centers nearest to $v$, represented by the probability distribution $\operatorname{pr}(\alpha, v)$. The $\alpha$-cluster-variance $\Psi(\alpha)$ measures large discrepancies between personalized PageRank vectors for nodes $v$ and the overall stationary distribution $\pi$. If the PageRank-variance $\Phi(\alpha)$ is small, then the 'guesses' by using PageRank vectors for the centers of mass give a good 
upper bound for the $k$-means evaluation $\mu$ using PageRank distance, indicating the formation of clusters. If the cluster-variance $\Psi(\alpha)$ is large, then the centers of masses using the predictions from PageRank vectors are quite far from the stationary distribution, capturing a community structure. Thus, our goal is to find the appropriate $\alpha$ such that $\Phi(\alpha)$ is small but $\Psi(\alpha)$ is large.

For a specific set of centers of mass $C$, we use the following for an evaluative metric $\Psi_{\alpha}(C)$, suggesting the structural separation of the communities represented by centers in $C$ :

$\Psi_{\alpha}(C)=\sum_{c \in C} \operatorname{vol}\left(R_{c}\right)\left\|\operatorname{pr}(\alpha, c) D^{-1 / 2}-\pi D^{-1 / 2}\right\|^{2}=\sum_{c \in C} \operatorname{vol}\left(R_{c}\right) \operatorname{dist}_{\alpha}(c, \pi)^{2}$.

We remark that this measure is essentially the analog of $k$-means in terms of PageRank distance, and it has a similar flavor as a heuristic given by Dyer and Frieze [11] for the traditional center selection problem. The metrics $\mu(C)$ and $\Psi_{\alpha}(C)$ are designed to evaluate a specific set of clusters $C$, while the measures $\Phi(\alpha)$ and $\Psi(\alpha)$ are well-suited to measure a graph's inherent clustered structure.

\section{The PageRank-Clustering Algorithms}

These evaluative measures give us a way to evaluate a set of community centers, leading to the PageRank-Clustering algorithms presented here. The problem of finding a set of $k$ centers minimizing $\mu(C)$ is then reduced to the problem of minimizing $\Phi(\alpha)$ while $\Psi(\alpha)$ is large for appropriate $\alpha$. In particular, for a special class of graphs which consist of $k$ clusters of vertices where each cluster has a bounded Cheeger ratio, the center selection algorithm is guaranteed to be successful with high probability.

A natural question is to find the appropriate $\alpha$ for a given graph, if such $\alpha$ exists and if the graph is clusterable. A direct method is by computing the variance metrics for a sample of $\alpha$ and narrowing down the range for $\alpha$ using binary search. Here, we give a systematic method for determining the existence of an appropriate $\alpha$ and finding its value is by differentiating $\Phi(\alpha)$, and finding roots $\alpha$ satisfying $\Phi^{\prime}(\alpha)=0$. It is not too difficult to compute that the derivative of $\Psi$ satisfies

$$
\Phi^{\prime}(\alpha)=\frac{1-\alpha}{\alpha^{3}}\left(\left\|g_{v}(\alpha) D^{-1 / 2}\right\|^{2}-2\left\langle g_{v}(\alpha), \operatorname{pr}\left(\alpha, g_{v}(\alpha)\right) D^{-1}\right\rangle\right)
$$

where $g_{v}(\alpha)=\operatorname{pr}(\alpha, \operatorname{pr}(\alpha, v)(I-W))$. Here, we give two versions of the clustering algorithm. For the sake of clarity, the first PageRank clustering algorithm uses exact PageRank vectors without approximation. The second PageRank clustering algorithm allows for the use of approximate PageRank vectors as well as approximate PageRank-variance and cluster-variance for faster performance.

We can further reduce the computational complexity by using approximate PageRank vectors in algorithm PageRank-ClusteringB.

We remark that by using the sharp approximate PageRank algorithm in [10], the error bound $\delta$ for PageRank can be set to be quite small since the time 


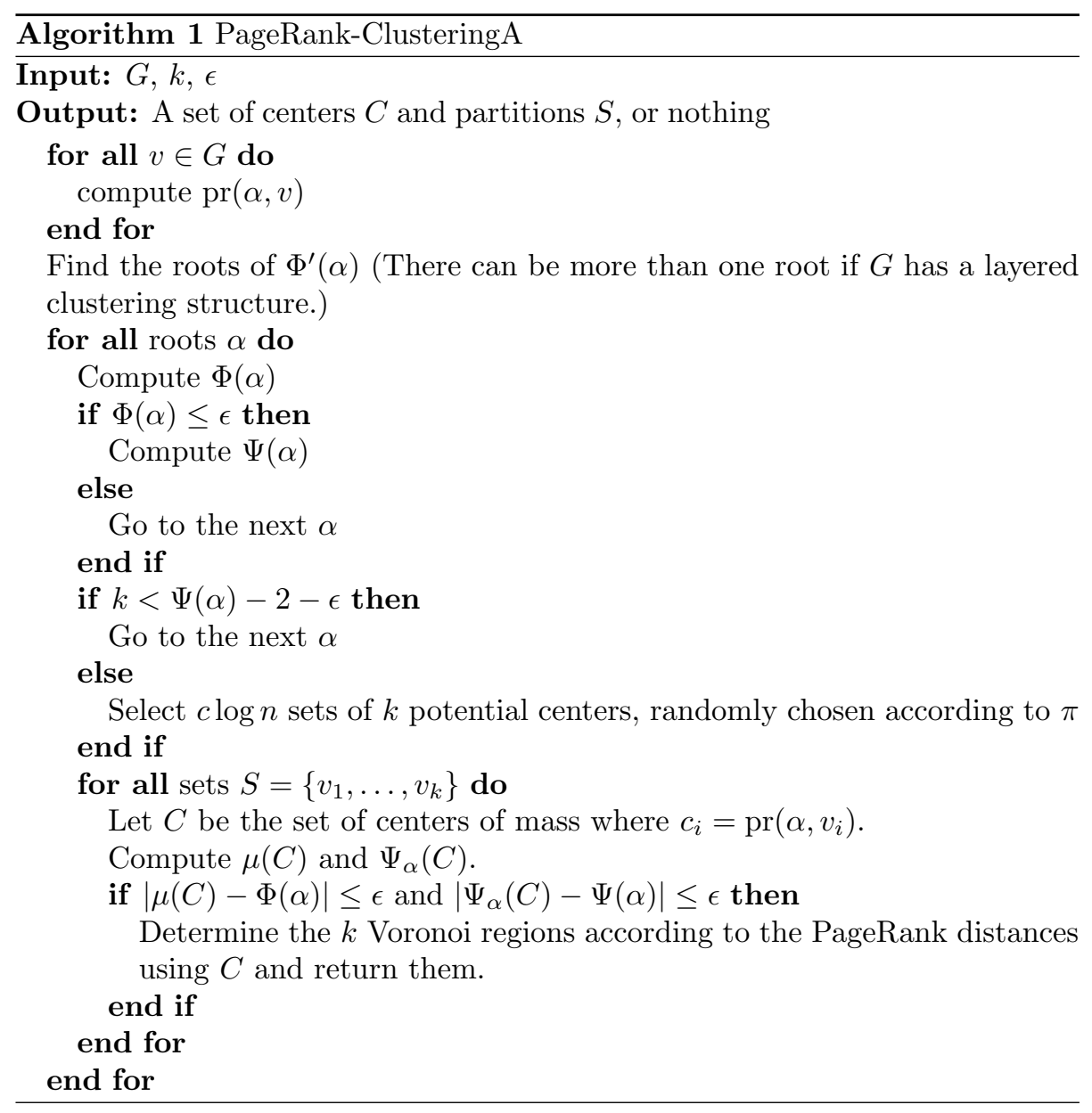




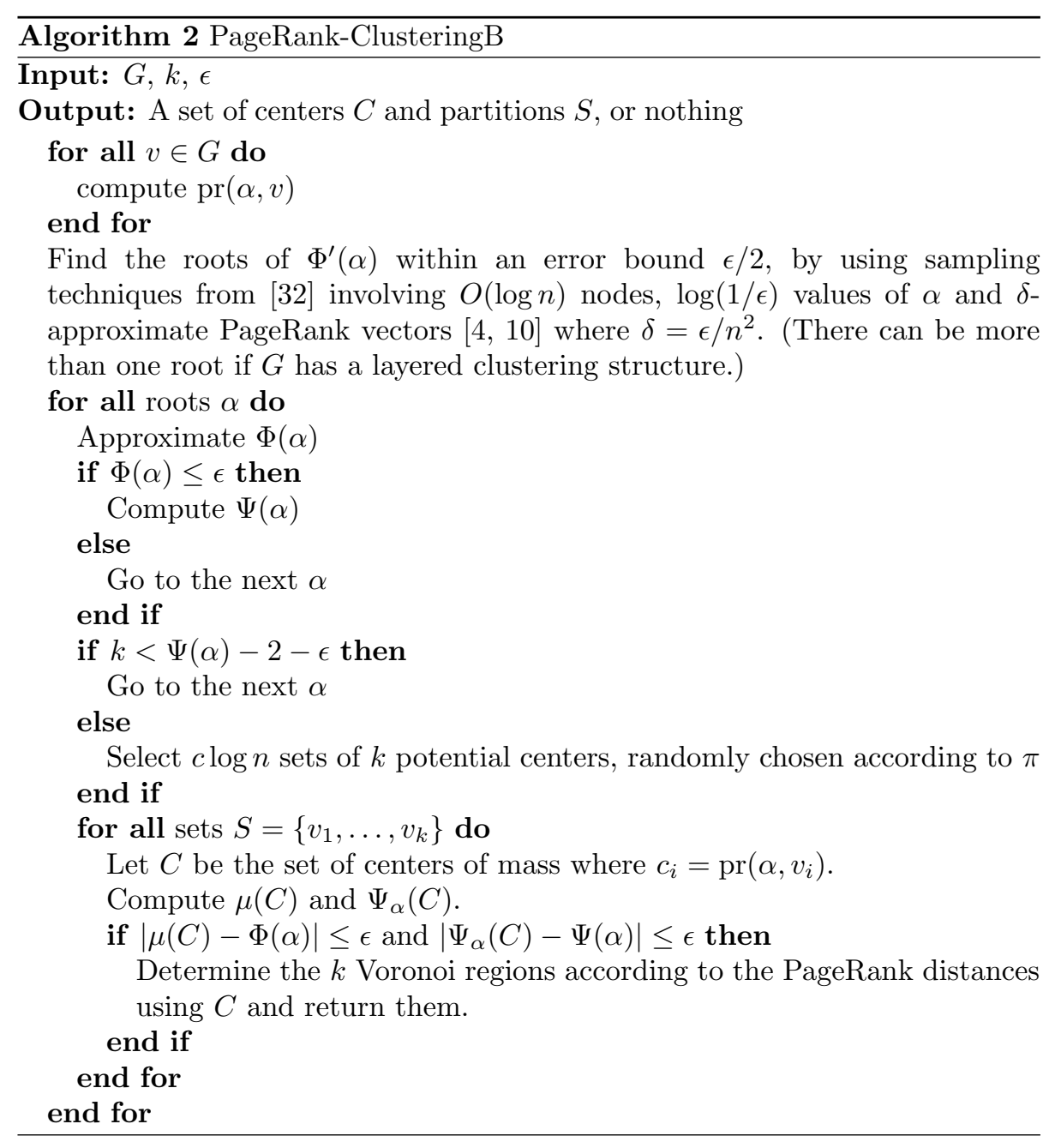


complexity is proportional to $\log (1 / \delta)$. If we choose $\delta$ to be a negative power of $n$ such as $\delta=\epsilon / n^{2}$, then approximate PageRank vectors lead to sharp estimates for $\Phi$ and $\Phi^{\prime}$ within an error bound of $\epsilon$. Thus for graphs with $k$ clusters, the PageRank-ClusteringB algorithm will terminate after approximating the roots of $\Phi^{\prime}, O(k \log n)$ approximations of $\mu$ and $\Psi_{\alpha}$ and $O(n)$ approximate PageRank computations. By using approximation algorithms using sampling, this can be done quite efficiently.

We also note that there might be no clustering output if the conditions set within the algorithms are not satisfied. Indeed, there exist graphs that inherently do not have a $k$-clustered structure within the error bound that we set for $\epsilon$. Another reason for no output is the probabilistic nature of the above sampling method. We will provide evidence to the correctness of the above algorithm by showing that, with high probability, a graph with a $k$-clustered structure will have outputs that capture its clusters in a feasible manner which we will specify further.

We say a graph $G$ is $(k, h, \beta, \epsilon)$-clusterable if the vertices of $G$ can be partitioned into $k$ parts so that:

1. Each part $S_{i}$ has Cheeger ratio at most $h$,

2. each $S_{i}$ has volume at least $\beta \operatorname{vol}(G) / k$ for some constant $\beta$, and

3. for each $S_{i}$, any subset $S_{i}^{\prime} \subset S_{i}$, with $\operatorname{vol}\left(S_{i}^{\prime}\right) \leq(1-\epsilon) \operatorname{vol}\left(S_{i}\right)$, has its Cheeger ratio at least $c \sqrt{h \log n}$ where $c=8 \sqrt{\beta / k} / \epsilon$.

We will provide evidence for the correctness of PageRank-ClusteringA by proving the following theorem:

Theorem 1. Suppose a graph $G$ has an $(k, h, \beta, \epsilon)$-clustering and $\alpha, \epsilon \in(0,1)$ satisfy $\epsilon \geq h k /(2 \alpha \beta)$. Then with high probability, PageRank-ClusteringA returns a set $C$ of $k$ centers with $\Phi(\alpha) \leq \epsilon, \Psi(C)>k-2-\epsilon$, and the $k$ clusters are near optimal according to the PageRank $k$-means measure $\mu$ with an additive error term $\epsilon$.

\section{Several facts about PageRank}

Before proceeding to show that the PageRank-clustering algorithms are effective for treating clusterable graphs, we will first establish some useful tools for analyzing PageRank vectors. These tools concern the diffusion of PageRank vectors in a subset of nodes with small Cheeger ratio. Before we examine a general mixing inequality involving PageRank vectors, first we consider a diffusion lower bound which is a slightly modified version of the results in [4].

Lemma 1. [4] For any set $S$ and any constants $\alpha, \delta$ in $(0,1]$, there is a subset $S_{\alpha} \subseteq S$ with volume $\operatorname{vol}\left(S_{\alpha}\right) \geq(1-\delta) \operatorname{vol}(S)$ such that for any vertex $v \in S_{\alpha}$, the PageRank vector $\operatorname{pr}(\alpha, v)$ satisfies

$$
[\operatorname{pr}(\alpha, v)](S) \geq 1-\frac{h(S)}{2 \alpha \delta} .
$$


We use the notation that for a function $f: V \rightarrow \mathbb{R}, f(S)=\sum_{v \in S} f(v)$ for $S \subseteq V$. For a positive real value $x$, we define

$$
f(x)=\max \left\{\sum_{v} \frac{\beta_{v}}{d_{v}} f(v): \sum_{v} \beta_{v}=x, 0 \leq \beta_{v} \leq d_{v}\right\} .
$$

This leads to many nice properties of $f$ including, for example that $f$ is concave and $f(\operatorname{vol}(S)) \geq f(S)$ (see $[4,23])$.

Lemma 2. For any set $S$ and any constants $\alpha, \delta$ in $(0,1]$, there is a subset $S_{\alpha} \subseteq S$ with volume $\operatorname{vol}\left(S_{\alpha}\right) \geq(1-\delta) \operatorname{vol}(S)$ such that for any vertex $v \in S_{\alpha}$, the PageRank vector $\operatorname{pr}(\alpha, \operatorname{pr}(\alpha, v))$ satisfies

$$
[\operatorname{pr}(\alpha, \operatorname{pr}(\alpha, v))](S) \geq 1-\frac{h(S)}{\alpha \delta} .
$$

Proof. The proof is quite similar to that in [4]. Let $\chi_{S}$ denote the function of $S$ which assumes the value $\chi_{S}(x)=d_{v} / \operatorname{vol}(S)$ if $x \in S$ and 0 otherwise. First we wish to show:

$$
\left[\operatorname{pr}\left(\alpha, \operatorname{pr}\left(\alpha, \chi_{S}\right)\right)\right](\bar{S}) \leq h(S) \frac{1-\alpha}{\alpha} .
$$

During a single step from $\operatorname{pr}\left(\alpha, \operatorname{pr}\left(\alpha, \chi_{S}\right)\right)$ to $\operatorname{pr}\left(\alpha, \operatorname{pr}\left(\alpha, \chi_{S}\right)\right) W$, the amount of probability that moves from $S$ to $\bar{S}$ is bounded from above by

$$
\left[\operatorname{pr}\left(\alpha, \operatorname{pr}\left(\alpha, \chi_{S}\right)\right) W\right](\bar{S}) \leq\left[\operatorname{pr}\left(\alpha, \operatorname{pr}\left(\alpha, \chi_{S}\right)\right)\right](\bar{S})+\frac{1}{2}\left[\operatorname{pr}\left(\alpha, \operatorname{pr}\left(\alpha, \chi_{S}\right)\right)\right](|\delta S|)
$$

where $\delta(S)$ denotes the edge boundary of $S$ consisting of edges leaving $S$. By using the definition of PageRank, we obtain

$$
\begin{aligned}
{\left[\operatorname{pr}\left(\alpha, \operatorname{pr}\left(\alpha, \chi_{S}\right)\right)\right](\bar{S}) } & =\alpha\left[\operatorname{pr}\left(\alpha, \chi_{S}\right)\right](\bar{S})+(1-\alpha)\left[\operatorname{pr}\left(\alpha, \operatorname{pr}\left(\alpha, \chi_{S}\right)\right) W\right](\bar{S}) \\
& \leq \frac{1-\alpha}{2} h(S)+(1-\alpha)\left[\operatorname{pr}\left(\alpha, \operatorname{pr}\left(\alpha, \chi_{S}\right)\right) W\right](\bar{S})
\end{aligned}
$$

by using Theorem 4 in [4] (inequality (8), specifically). From (2), we have

$$
\begin{aligned}
& {\left[\operatorname{pr}\left(\alpha, \operatorname{pr}\left(\alpha, \chi_{S}\right)\right) W\right](\bar{S}) } \\
= & \frac{1-\alpha}{2} h(S)+(1-\alpha)\left[\operatorname{pr}\left(\alpha, \operatorname{pr}\left(\alpha, \chi_{S}\right)\right)\right](\bar{S})+\frac{1-\alpha}{2}\left[\operatorname{pr}\left(\alpha, \operatorname{pr}\left(\alpha, \chi_{S}\right)\right)\right](|\delta S|) .
\end{aligned}
$$

This implies

$$
\left[\operatorname{pr}\left(\alpha, \operatorname{pr}\left(\alpha, \chi_{S}\right)\right)\right](\bar{S})=\frac{1-\alpha}{2 \alpha} h(S)+\frac{1-\alpha}{2 \alpha}\left[\operatorname{pr}\left(\alpha, \operatorname{pr}\left(\alpha, \chi_{S}\right)\right)\right](|\delta S|) .
$$

Now we use the monotonicity property from Lemma 4 in [4]; we have

$$
\begin{aligned}
{\left[\operatorname{pr}\left(\alpha, \operatorname{pr}\left(\alpha, \chi_{S}\right)\right)\right](|\delta S|) } & \leq\left[\operatorname{pr}\left(\alpha, \chi_{S}\right)\right](|\delta(S)|) \\
& \leq \chi_{S}(|\delta(S)|) \\
& =\frac{|\delta(S)|}{\operatorname{vol}(S)} \\
& =h(S) .
\end{aligned}
$$


Thus we have

$$
\left[\operatorname{pr}\left(\alpha, \operatorname{pr}\left(\alpha, \chi_{S}\right)\right)\right](\bar{S}) \leq \frac{1-\alpha}{\alpha} h(S) .
$$

To complete the proof, let $S_{\alpha}$ denote the set of vertices $v$ in $S$ satisfying

$$
[\operatorname{pr}(\alpha, \operatorname{pr}(\alpha, v))](\bar{S}) \leq \frac{h(S)}{\alpha \delta} .
$$

Let $v$ be a vertex chosen randomly from the distribution $d_{v} / \operatorname{vol}(S)$, and define the random variable $X=[\operatorname{pr}(\alpha, \operatorname{pr}(\alpha, v))](\bar{S})$. The linearity property of PageRank vectors implies that

$$
E(X)=\left[\operatorname{pr}\left(\alpha, \operatorname{pr}\left(\alpha, \chi_{S}\right)\right)\right](\bar{S}) \leq \frac{1-\alpha}{\alpha} h(S) \leq \frac{h(S)}{\alpha} .
$$

Applying Markov's inequality, we have

$$
\operatorname{Pr}\left[v \notin S_{\alpha}\right] \leq \operatorname{Pr}[X \geq E[X] / \delta] \leq \delta .
$$

This completes the proof of Lemma 2 .

We will also need the quantitative estimates for PageRank vectors restricted to a subset $S$ of vertices. By considering submatrices $W_{S}$ restricted to rows and columns associated with vertices in $S$, we can define the Dirichlet PageRank $\operatorname{pr}_{S}(\alpha, s)$, for a seed vector defined on $S$ and $0 \leq \alpha<1$ satisfying:

$$
\operatorname{pr}_{S}(\alpha, \vec{s})=\alpha \vec{s}+(1-\alpha) \operatorname{pr}_{S}(\alpha, \vec{s}) W_{S} .
$$

When $\alpha$ is appropriately chosen, the Dirichlet PageRank is a good estimate of PageRank vectors. Lemma 5 and Theorem 6 in [8] can be rewritten as follows.

Lemma 3. [8] Suppose a subset $S$ of vertices has its Cheeger ratio $h(S)$ satisfying $\epsilon \geq \frac{(1-\alpha) h(T)}{2 \alpha}$, for positive values $\alpha, \epsilon$. Then $\operatorname{pr}_{S}$ satisfies the following: For any $R \subseteq S$, There is a subset $T \subseteq S$ with $\operatorname{vol}(T) \geq(1-\delta) \operatorname{vol}(S)$, so that for every $v$ in $T$ we have

$$
[\operatorname{pr}(\alpha, v)](R)-\left[\operatorname{pr}_{S}(\alpha, v)\right](R) \leq \sqrt{\frac{\epsilon}{\delta}} .
$$

For a probability distribution $f: V \rightarrow \mathbb{R}$ and a real value $x$, we define the Cheeger ratio $h_{f}(x)$ of $f$ up to $x$ as follows: We order the vertices $v_{1}, v_{2}, \ldots, \ldots$ from highest to lowest probability-per-degree, so that $p\left(v_{i}\right) / d\left(v_{i}\right) \geq$ $p\left(v_{i+1}\right) / d\left(v_{i+1}\right)$. This produces a collection of sets, called the segment subsets, with one set $T_{j}^{f}=\left\{v_{1}, \ldots, v_{j}\right\}$ for each $j \leq n$. For a positive value $x \leq \operatorname{vol}(G)$, we define

$$
\begin{aligned}
& h_{f}(x)=\max \left\{h\left(T_{j}^{f}\right): j \text { satisfies } \operatorname{vol}\left(T_{j}^{f}\right) \leq x\right\} . \\
& h_{f}^{*}(x)=\max \left\{h\left(T_{j}^{f}\right): j \text { satisfies } \operatorname{vol}\left(T_{j}^{f}\right) \leq x\left(1+h_{f}(x)\right)\right\} .
\end{aligned}
$$


Lemma 4. [4] For a vertex in $G$, any constant $\alpha$ in $(0,1]$ and non-negative integer $t$, the PageRank vector $\operatorname{pr}(\alpha, v)$ satisfies the following:

$$
[\operatorname{pr}(\alpha, v)](T)-\pi(T) \leq \alpha t+\sqrt{\operatorname{vol}(T)}\left(1-\frac{\phi^{2}}{8}\right)^{t}
$$

where $\phi$ is the Cheeger ratio $h_{f}^{*}(\operatorname{vol}(T))$ with $f=\operatorname{pr}(\alpha, v)$.

Lemma 5. For subsets $S, T$ of vertices in $G$ with $\operatorname{vol}(S), \operatorname{vol}(T) \leq \operatorname{vol}(G) / 2$, any constant $\alpha$ in $(0,1]$ and non-negative integer $t$, the Dirichlet PageRank vector $\operatorname{pr}_{S}(\alpha, v)$ for any vertex $v$ in $S$ satisfies the following:

(i)

$$
\left[\operatorname{pr}_{S}(\alpha, v)\right](T)-\left[\operatorname{pr}_{S}\left(\alpha, \operatorname{pr}_{S}(\alpha, v)\right)\right](T) \leq \alpha t+\sqrt{\operatorname{vol}(T)}\left(1-\frac{\phi^{2}}{8}\right)^{t}
$$

where $\phi$ is the Cheeger ratio $h_{f}^{*}(\operatorname{vol}(T))$ with $f=\operatorname{pr}_{S}(\alpha, v)-\operatorname{pr}_{S}\left(\alpha, \operatorname{pr}_{S}(\alpha, v)\right)$. (ii)

$$
\left[\operatorname{pr}_{S}\left(\alpha, \operatorname{pr}_{S}(\alpha, v)\right)\right](T)-\left[\operatorname{pr}_{S}(\alpha, v)\right](T) \leq \alpha t+\sqrt{\operatorname{vol}(T)}\left(1-\frac{\phi^{\prime 2}}{8}\right)^{t}
$$

where $\phi^{\prime}$ is the Cheeger ratio $h_{f}^{\prime *}(\operatorname{vol}(T))$ with $f^{\prime}=\operatorname{pr}_{S}\left(\alpha, \operatorname{pr}_{S}(\alpha, v)\right)-\operatorname{pr}_{S}(\alpha, v)$. (iii) For two vertices $u$ and $v$,

$$
\left[\operatorname{pr}_{S}(\alpha, u)\right](T)-\left[\operatorname{pr}_{S}(\alpha, v)\right](T) \leq \alpha t+\sqrt{\operatorname{vol}(T)}\left(1-\frac{\phi^{\prime \prime 2}}{8}\right)^{t}
$$

where $\phi^{\prime \prime}$ is the Cheeger ratio $h_{f}^{\prime \prime *}(\operatorname{vol}(T))$ with $f^{\prime \prime}=\operatorname{pr}_{S}(\alpha, u)-\operatorname{pr}_{S}(\alpha, v)$.

Proof. We prove by induction on $t$ for (i). For $t=0$, it holds. Suppose the above inequality holds for some $t \geq 0$. Let $x$ denote $\operatorname{vol}(T)$. We use Lemma 3 of [4] and apply the same method using the concavity of $f$ to obtain:

$$
\begin{aligned}
{\left[\operatorname{pr}_{S}(\alpha, v)\right](T)-\left[\operatorname{pr}_{S}\left(\alpha, \operatorname{pr}_{S}(\alpha, v)\right)\right](T) } & =f(T) \\
\leq & \alpha+(1-\alpha)[f W](T) \\
\leq & \alpha+(1-\alpha)\left(\frac{1}{2} f(x-\phi x)+\frac{1}{2} f(x+\phi x)\right) \\
\leq & \alpha+\left(\frac{1}{2} f(x-\phi x)+\frac{1}{2} f(x+\phi x)\right)
\end{aligned}
$$

Using the induction assumption, we have

$$
\begin{aligned}
f(T) & \leq \alpha(t+1)+\frac{1}{2}(\sqrt{x-\phi x}+\sqrt{x+\phi x})\left(1-\frac{\phi^{2}}{8}\right)^{t} \\
& \leq \alpha(t+1)+\sqrt{x}\left(1-\frac{\phi^{2}}{8}\right)^{t+1} .
\end{aligned}
$$

This proves (i). We omit the proofs for (ii) and (iii) which can be done in a similar way. The proof of Lemma 5 is complete. 


\section{Analyzing PageRank Clustering Algorithms}

In this section, we consider an $(h, k, \beta, \epsilon)$-clusterable graph $G$, with the following condition:

$$
\epsilon \geq \frac{h k}{2 \alpha \beta} .
$$

Lemma 1 implies that in a cluster $R$ of $G$, most of the vertices $u$ in $R$ have $\operatorname{pr}(\alpha, u)(S) \geq 1-\epsilon /(2 k)$. This fact is essential in the subsequent proof that $\Psi(\alpha) \geq k-2-\epsilon$.

We proceed with a series of lemmas that show that if $G$ is $(h, k, \beta, \epsilon)$ clusterable, there is an $\alpha$ for which $\Phi(\alpha)$ is small and $\Psi(\alpha)$ is large, corresponding to a set of centers chosen from the core of the partitions.

Lemma 6. If a graph $G$ can be partitioned into $k$ clusters having Cheeger ratio at most $h$ and $\epsilon \geq h k /(2 \alpha \beta)$, then $\Psi(\alpha) \geq k-2-\epsilon$.

Proof. Let $S_{1}, \ldots, S_{k}$ be a partition of $G$ into $k$ clusters satisfying the theorem conditions. Then, by definition of $\Psi$,

$$
\begin{aligned}
\Psi(\alpha) & =\sum_{v \in V} d_{v}\left\|\operatorname{pr}(\alpha, \operatorname{pr}(\alpha, v)) D^{-1 / 2}-\pi D^{-1 / 2}\right\|^{2} \\
& =\sum_{i=1}^{k} \sum_{v \in S_{i}} d_{v}\left\|\operatorname{pr}(\alpha, \operatorname{pr}(\alpha, v)) D^{-1 / 2}-\pi D^{-1 / 2}\right\|^{2} \\
& =\sum_{i=1}^{k} \sum_{v \in S_{i}} d_{v} \sum_{x \in V}\left(\operatorname{pr}(\alpha, \operatorname{pr}(\alpha, v)) D^{-1 / 2}(x)-\pi D^{-1 / 2}(x)\right)^{2} \\
& \geq \sum_{i=1}^{k} \sum_{v \in S_{i}} d_{v} \sum_{x \in S_{i}}\left(\operatorname{pr}(\alpha, \operatorname{pr}(\alpha, v)) D^{-1 / 2}(x)-\pi D^{-1 / 2}(x)\right)^{2} \\
& =\sum_{i=1}^{k} \sum_{v \in S_{i}} d_{v} \sum_{x \in S_{i}}\left(\operatorname{pr}(\alpha, \operatorname{pr}(\alpha, v)) D^{-1 / 2}(x)-\pi D^{-1 / 2}(x)\right)^{2} \sum_{x \in S_{i}} \frac{d_{x}}{\operatorname{vol}\left(S_{i}\right)} \\
& =\sum_{i=1}^{k} \sum_{v \in S_{i}} d_{v} \sum_{x \in S_{i}} \frac{1}{d_{x}}(\operatorname{pr}(\alpha, \operatorname{pr}(\alpha, v))(x)-\pi(x))^{2} \sum_{x \in S_{i}} \frac{d_{x}}{\operatorname{vol}\left(S_{i}\right)} .
\end{aligned}
$$


Using the Cauchy-Schwarz inequality and then Lemma 2, we have

$$
\begin{aligned}
\Psi(\alpha) & \geq \sum_{i=1}^{k} \sum_{v \in S_{i}} \frac{d_{v}}{\operatorname{vol}\left(S_{i}\right)}\left(\sum_{x \in S_{i}}(\operatorname{pr}(\alpha, \operatorname{pr}(\alpha, v))(x)-\pi(x))\right)^{2} \\
& \geq \sum_{i=1}^{k} \sum_{v \in S_{i}} \frac{d_{v}}{\operatorname{vol}\left(S_{i}\right)}\left(1-\frac{\epsilon}{2}-\frac{\operatorname{vol}\left(S_{i}\right)}{\operatorname{vol}(G)}\right)^{2} \\
& =\sum_{i=1}^{k}\left(1-\frac{\epsilon}{2}-\frac{\operatorname{vol}\left(S_{i}\right)}{\operatorname{vol}(G)}\right)^{2} \\
& \geq \frac{1}{k}\left(\sum_{i=1}^{k}\left(1-\frac{\epsilon}{2}-\frac{\operatorname{vol}\left(S_{i}\right)}{\operatorname{vol}(G)}\right)\right)^{2} \\
& =\frac{1}{k}\left(k-1-\frac{\epsilon}{2}\right)^{2} \\
\geq & k-2-\epsilon .
\end{aligned}
$$

We have shown that if $G$ has a clustered structure, then there is an $\alpha$ for which $\Psi(\alpha)$ is large. We will also show that our algorithm will also yield $\Phi(\alpha) \leq$ $\epsilon$.

Lemma 7. If $G$ is $(k, h, \beta, \epsilon)$-clusterable, then we have $\Phi(\alpha) \leq \epsilon$.

Proof. The proof follows from preceding lemmas. Within each cluster $S$ of $G$, we first use Lemma 2 which implies there is a subset $S^{\prime}$ of $S$ such that $[\operatorname{pr}(\alpha, v)](S) \geq 1-\epsilon / k$ and $\operatorname{vol}\left(S^{\prime}\right) \geq(1-\delta) \operatorname{vol}(S)$ since $S$ has Cheeger ratio at most $h$.

We can apply Lemma 3 so that we can approximate PageRank vectors $\operatorname{pr}(\alpha, v)$ by the Dirichlet PageRank vectors $\operatorname{pr}_{S}(\alpha, v)$.

From the definition of an $(k, h, \beta, \epsilon)$-clusterable graph, each subset $T$ of $S$ has Cheeger ratio at least $c \sqrt{h \log n}$. This allows us to use Lemma 5 for any segment subset $T_{j}^{f}$ (as defined in (3) with volume at most $(1-\epsilon / 2) \operatorname{vol}(S)$ defined by the function $f$ as in Lemma 5 . Together we have that, for any subset $R \subset S$ with $\operatorname{vol}(R) \leq(1-\epsilon / 2) \operatorname{vol}(S)$,

$$
\begin{aligned}
\mid[\operatorname{pr}(\alpha, v)](R)-[\operatorname{pr}(\alpha, \operatorname{pr}(\alpha, v)](R) \mid & \leq \alpha t+\sqrt{n} e^{-\left(c^{2} t h \log n\right) / 8} \\
& \leq \frac{\epsilon}{4}
\end{aligned}
$$

by the assumption that $c=8 \sqrt{\beta / k} / \epsilon$, and choosing $t=1 /\left(h c^{2}\right)$. This implies that for any subset $R \subset S$ and any vertex $v$, we have

$$
\mid[\operatorname{pr}(\alpha, v)](R)-\left[\operatorname{pr}(\alpha, \operatorname{pr}(\alpha, v)](R) \mid \leq \frac{\epsilon}{2}\right.
$$


Thus the total variation distance between the two PageRank vectors is:

$$
\Delta_{T V}(\alpha)=\max _{v} \max _{R \subseteq S}[\operatorname{pr}(\alpha, v)-\operatorname{pr}(\alpha, \operatorname{pr}(\alpha, v))](R) \leq \frac{\epsilon}{2} .
$$

Note that $\sqrt{\Phi(\alpha)}$ is just the so-called $\chi$-square distance $\Delta_{\chi}$. Using the same technique as in [2], we have

$$
\Delta_{T V} \leq \Delta_{\chi} \leq \sqrt{1-\left(1-2 \Delta_{T V}\right)^{2}}
$$

Thus, we conclude that $\Phi(\alpha) \leq \epsilon$ as desired.

We will also show that the sampling methods that PageRank-ClusteringA does will ensure that with high probability, the cluster centers $\left\{c_{1}, \ldots, c_{k}\right\}$ will include one from the core of each of $k$ partitions in a clusterable graph:

Lemma 8. Suppose $G$ is $(h, k, \beta, \epsilon)$-clusterable, and $c \log n$ sets of $k$ potential centers are chosen from $G$ according to the stationary distribution $\pi$, where $c$ is some absolute constant. With probability $1-o(1)$, at least one set will contain one vertex from the core of each of the $k$ clusters.

Proof. Let $S_{1}, \ldots, S_{k}$ be a partition of $(h, k, \beta, \epsilon)$-clusterable $G$, and let $S_{i}^{\prime}$ be the core of $S_{i}$. Suppose vertices $C=\left\{c_{1}, \ldots, c_{k}\right\}$ are chosen randomly according to $\pi$, and let $E(C)$ be the event that each $c_{i} \in S_{i}^{\prime}$. Then, we have

$$
\begin{aligned}
\operatorname{Pr}[E(C)] & \geq \prod_{i=1}^{k} \operatorname{Pr}\left[c_{i} \in S_{i}^{\prime}\right] \\
& =\prod_{i=1}^{k} \frac{\operatorname{vol}\left(S_{i}^{\prime}\right)}{\operatorname{vol}(G)} \\
& \geq \prod_{i=1}^{k} \frac{(1-\epsilon) \operatorname{vol}\left(S_{i}^{\prime}\right)}{\operatorname{vol}(G)} \\
& \geq \prod_{i=1}^{k} \frac{(1-\epsilon) \beta \operatorname{vol}(G)}{k \operatorname{vol}(G)} \\
& =\left(\frac{\beta(1-\epsilon)}{k}\right)^{k} .
\end{aligned}
$$

If $c \log n$ sets $C_{1}, \ldots, C_{c \log n}$ of $k$ centers are sampled independently, the probability that at least one contains each $c_{i} \in S_{i}^{\prime}$ is:

$$
\begin{aligned}
\operatorname{Pr}\left[E\left(C_{1}\right) \vee \cdots \vee E\left(C_{c \log n}\right)\right] & \geq 1-\prod i=1^{c \log n} \operatorname{Pr}\left[\neg E\left(C_{i}\right)\right] \\
& =1-\prod i=1^{c \log n}\left(1-\operatorname{Pr}\left[E\left(C_{i}\right)\right]\right) \\
& \geq 1-\left(1-\left(\frac{\beta(1-\epsilon)}{k}\right)^{k}\right)^{c \log n} \\
& =1-o(1) .
\end{aligned}
$$


This series of lemmas then leads to the proof of Theorem 1, showing the correctness of PageRank-ClusteringA.

Proof. We note that $\operatorname{pr}(0, s)=\pi$ and $\operatorname{pr}(1, s)=s$ for any distribution $s$. This implies that $\Phi(0)=\Phi(1)=\Psi(0)=0$ and $\Psi(1)=n-1$. It is not hard to check that $\Psi$ is an increasing function since $\Psi^{\prime}(\alpha)>0$ for $\alpha \in(0,1]$. The function of particular interest is $\Phi$. Since we wish to find $\alpha$ such that $\Phi$ is small, it suffices to check the roots of $\Phi^{\prime}$ for an $\alpha$ where $\Phi(\alpha)<\epsilon$, which our algorithm does. Such an $\alpha$ exists due to Lemma 3 .

Suppose $\alpha$ is a root of $\Phi^{\prime}$. To find $k$ clusters, we can further restrict ourselves to the case of $\Psi(\alpha) \geq k-2-\epsilon$ by Lemma 2 .

We note that by sampling $c \log n$ sets of $k$ vertices from $\pi$, for sufficiently large $c$, the values $\mu(C)$ and $\Psi(C)$ for one such random set of $k$ centers are close to $\Phi(\alpha)$ and $\Psi(\alpha)$, respectively, with high probability (exponentially decreasing depending on $c$ and $\beta$ ) by probabilistic concentration arguments. In this context, the upper bound $\epsilon$ for $\mu(C)$ implies that the set consisting of distributions $\operatorname{pr}(\alpha, c)$ for $c \in C$ serves well as the set of centers of mass. Thus, the resulting Voronoi regions using $C$ give the desired clusters. This proves the correctness of our clustering algorithm with high probability for $(k, h, \beta, \epsilon)$-clusterable graphs.

To illustrate PageRank-ClusteringB, we consider a dumbbell graph $U$ as an example. This graph $U$ has two complete graphs $K_{20}$ connected by a single edge, yielding a Cheeger ratio of $h \approx 0.0026$. Plotting $\Phi(\alpha)$ (Fig. 1) and its derivative (Fig. 2 ) shows that there is a local minimum near $\alpha \approx 0.018$. When $\Psi$ is large, many individual nodes have personalized PageRank vectors that differ greatly from the overall distribution. This indicates that there are many nodes that are more representative of a small cluster than the entire graph. By plotting $\Psi(\alpha)$ (Fig. 3) and its derivative (Fig. 4), we can see that there is a distinct inflection point in the plot of $\Psi$ for the dumbbell graph $U$ as well.

\section{A Graph Drawing Algorithm Using PageRank}

The visualization of complex graphs provides many computational challenges. Graphs such as the World Wide Web and social networks are known to exhibit ubiquitous structure, including power-law distributions, small-world phenomena, and a community structure $[1,7,14]$. With large graphs, it is easy for such intricate structures to be lost in the sheer quantity of the nodes and edges, which can result in drawings that reflect a network's size but not necessarily its structure.

Given a set of nodes $S$, we can extract communities around each node and determine the layout of the graph using personalized PageRank. The arrangement can be done using a force-based graph layout algorithm such as the KamadaKawai algorithm [21]. The goal is to capture local communities; we can do this by assigning edges $\{s, v\}$ for each $s \in S$ and $v \in V \backslash S$ with weight inversely 


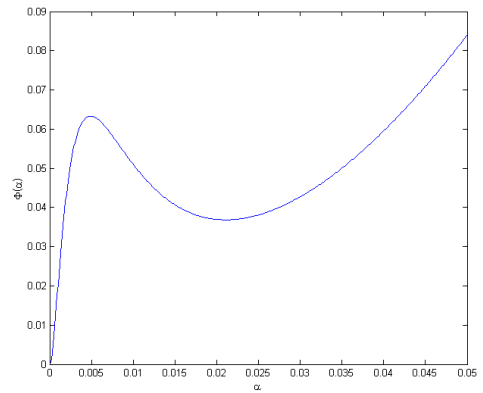

Figure 1: $\Phi(\alpha)$ for the dumbell graph $U$.

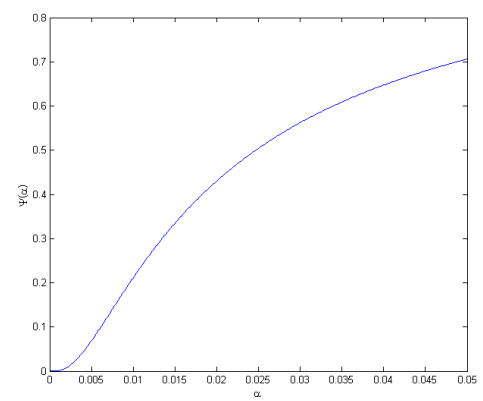

Figure 3: $\Psi(\alpha)$ for the dumbell graph $U$.

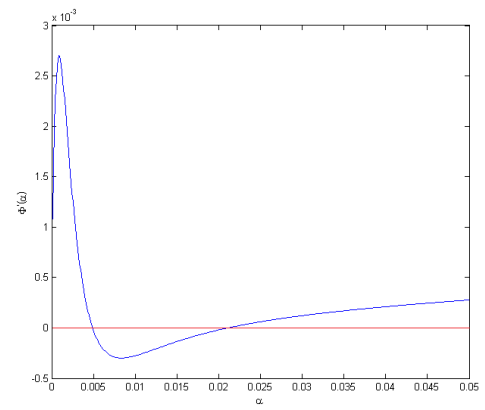

Figure 2: $\Phi^{\prime}(\alpha)$ for the dumbell graph $U$, with the line $y=0$ for reference.

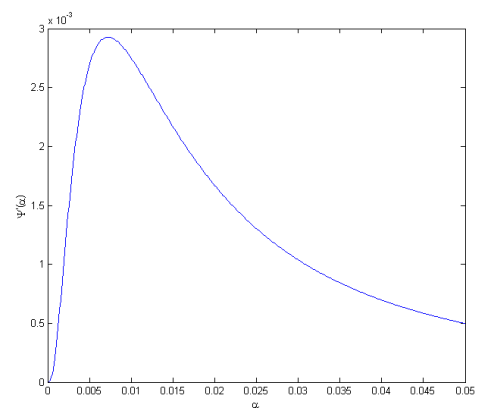

Figure 4: $\Psi^{\prime}(\alpha)$ for the dumbell graph $U$.

proportional to the personalized PageRank. This way, unrelated nodes with low PageRank will be forced to be distant, and close communities will remain close together. We also add edges $\left\{s, s^{\prime}\right\}$ for $s, s^{\prime} \in S$ with large weight to encourage separation of the individual communities. We use an implementation from Graphviz [16].

We note that because force-based algorithms are simulations, they do not guarantee the exact cluster structure, but we will illustrate that it works well in practice. Additionally, there are algorithms specifically designed for clustered graph visualization [12,31] and highlighting high-ranking nodes [5], but they impose a lot of artificial hierarchical structure onto the drawing and often require precomputing the clusters. Once we have a layout for all the nodes in the graph, we can partition them by using a Voronoi diagram. We compute the Voronoi diagram efficiently using Fortune's algorithm [15].

We tie together personalized PageRank and Voronoi diagrams in the algo- 
rithm PageRank-Display.

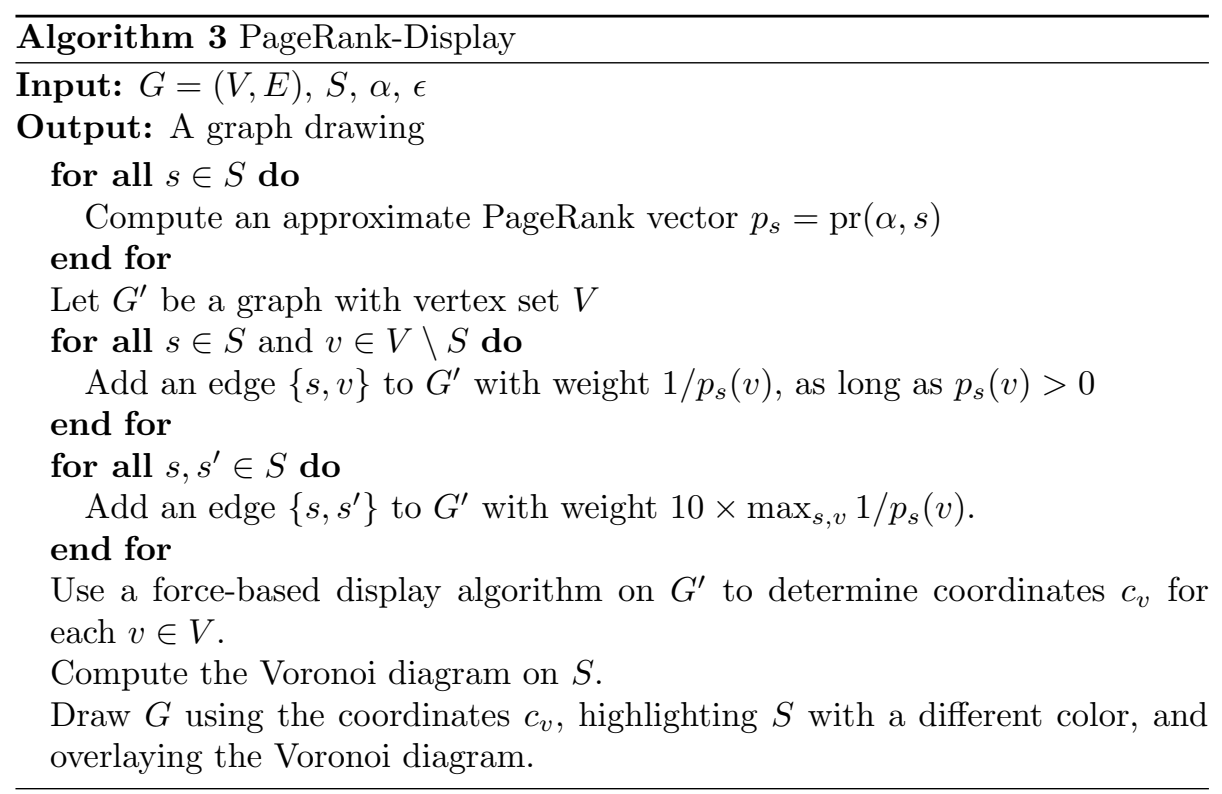

The jumping constant $\alpha$ is associated with the scale of the clustering. We can determine $\alpha$ either by trial and error or by optimizing $\Phi$ and $\Psi$ as in section 4. As long as $G$ is connected, the PageRank vector will be nonzero on every vertex. Using the algorithms from [4, 10], the approximation factor $\epsilon$ acts as a cutoff, and any node $v$ with PageRank less than $\epsilon d_{v}$ will be assigned zero. This is advantageous because the support of the approximate PageRank vector will be limited to the local community containing its seed. In PageRank-Display, we give weights to the edges equal to $1 / p_{s}(v)$, but this is problematic if $p_{s}(v)=0$. In that case, we omit the edge from $G^{\prime}$ entirely.

We remark that the selection of $\epsilon$ will influence the size of the local communities: the subset of nodes with nonzero approximate PageRank has volume at most $\frac{2}{(1-\alpha) \epsilon}$ (see [4]). This implies that a good selection of $\epsilon$ is $O\left(\frac{|S|}{(1-\alpha) \operatorname{vol}(G)}\right)$.

We also remark that the selection of $S$ is important. If $S$ contains vertices that are not part of communities or two nodes in the same community, then there will be no structure to display. In general, the selection of $S$ is similar to the geometric problem of finding a set of points with minimum covering radius, which can be intractable (see [18]). There are several algorithms that can automatically choose $S$, including PageRank-Clustering as presented here.

We used our algorithm to demonstrate and highlight the existence of local structure in two real-world datasets. The first dataset is a social network among 62 dolphins [24]. While the graph exhibits traditional network structure such as small-world phenomena, one can see in Figs. 5 and 6 that the dolphins can be divided into two communities, with just a few connected to both sides. 


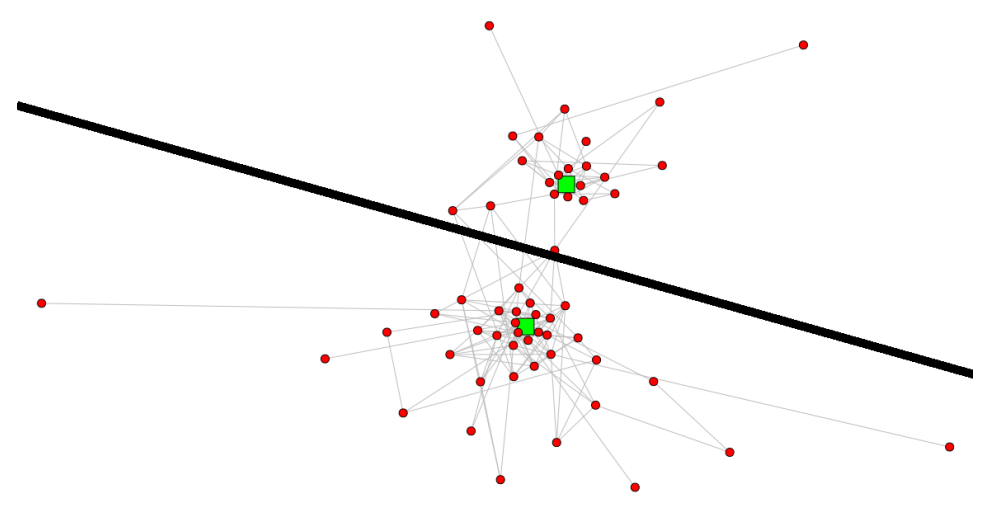

Figure 5: Results of PageRank-Display $(\alpha=0.03)$ on the dolphin social network [24], separating the dolphins into two communities.

Note that with larger $\alpha$, the far-flung nodes become more isolated, making the communities appear denser.

A more interesting example is shown in Figs. 7 and 8. The vertices represent 114 NCAA Division I American collegiate football teams, with edges connecting two teams if they played against each other during the 2000 football season. The league is divided into many smaller conferences of up to 12 teams; for each team, about half of its games are played against conference opponents, and the rest are played against nonconference teams. An appropriate selection of the 8 highlighted teams in Figs. 7 and 8 reveal a partition that separates their 8 respective conferences, and teams from the remaining conferences are placed on the periphery of the drawing. Here, the larger $\alpha$ is more effective as the PageRank is more concentrated near the community centers.

\section{References}

[1] R. Albert, A.-L. Barabási and H. Jeong. Diameter of the World Wide Web. Nature 401 (1999), 130-131.

[2] D. Aldous and J. Fill, Reversible Markov Chains and random graphs on graphs, monograph in preparation.

[3] R. Andersen and F. Chung. Detecting sharp drops in PageRank and a simplified local partitioning algorithm. Proceedings of the 4th International Conference Theory and Applications of Models of Computation (2007), 1-12.

[4] R. Andersen, F. Chung and K. Lang. Local graph partitioning using PageRank vectors. Proceedings of the 47 th Annual IEEE Symposium on Foundation of Computer Science (FOCS 2006), 475-486. 


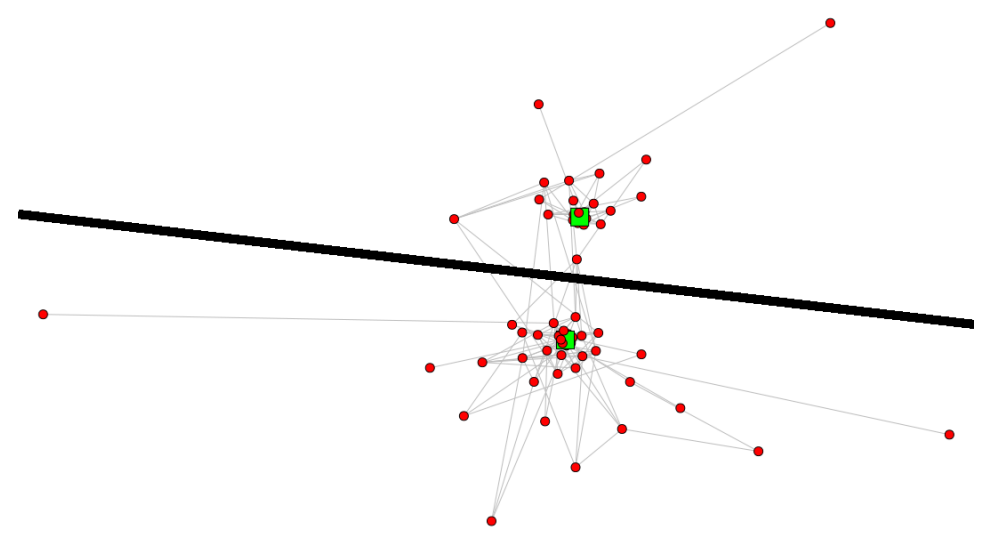

Figure 6: Results of PageRank-Display $(\alpha=0.3)$ on the dolphin social network [24], separating the dolphins into two communities.

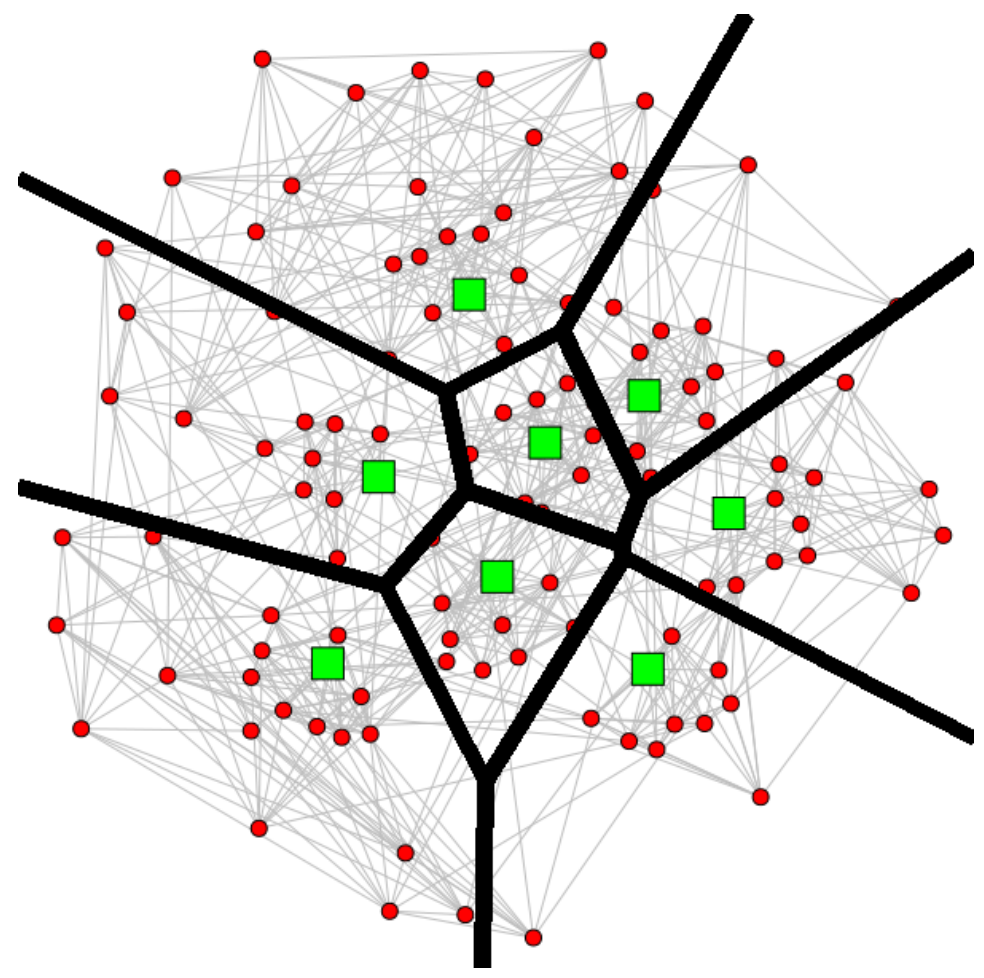

Figure 7: Results of PageRank-Display $(\alpha=0.1)$ on the football game network [17], highlighting 8 of the major collegiate conferences. 


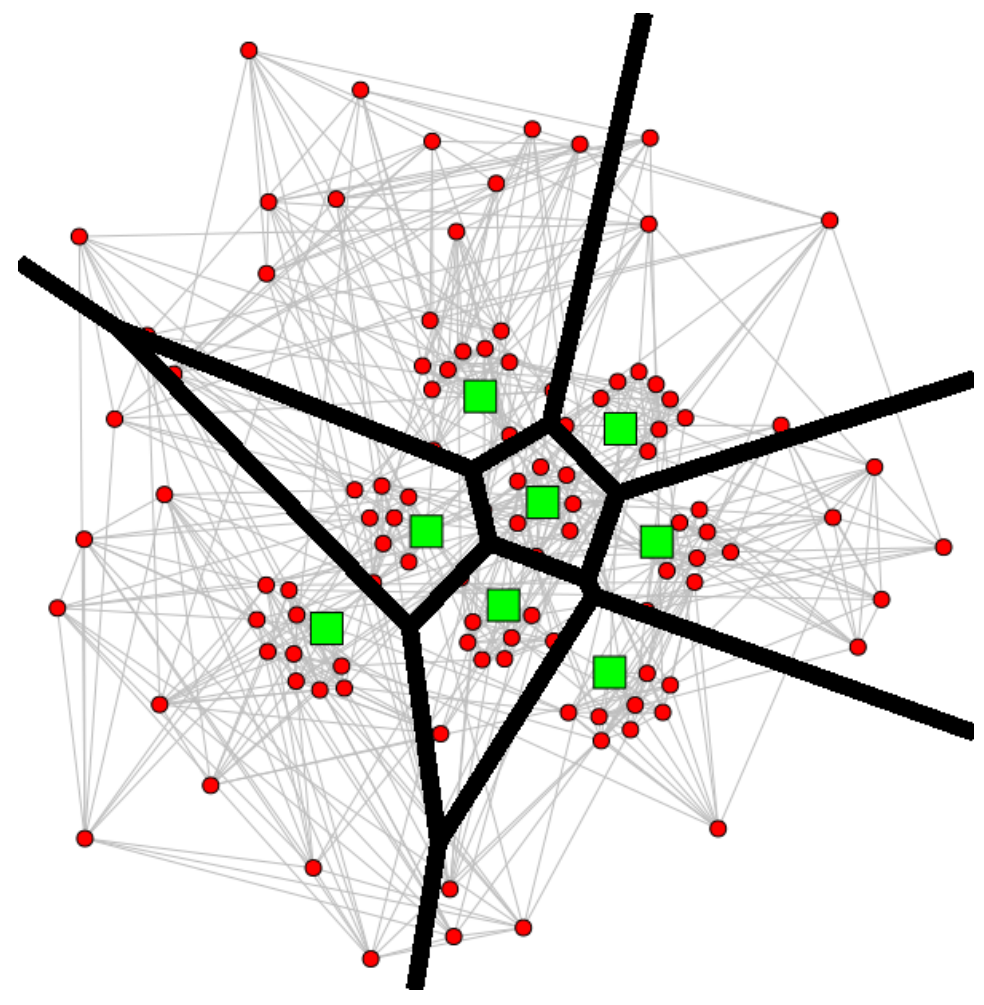

Figure 8: Results of PageRank-Display $(\alpha=0.3)$ on the football game network [17], highlighting 8 of the major collegiate conferences. 
[5] U. Brandes and S. Cornelsen. Visual ranking of link structures. Proceedings of the 7th International Workshop on Algorithms and Data Structures (2001), 222-233.

[6] S. Brin and L. Page. The anatomy of a large-scale hypertextual Web search engine. Computer Networks and ISDN Systems 30 (1998), 107-117.

[7] A. Broder, R. Kumar, F. Maghoul, P. Raghavan, S. Rajagopalan, R. Stata, A. Tomkins and J. Wiener. Graph structure in the Web. Computer Networks 33 (2000), 1-6.

[8] F. Chung, PageRank as a discrete Green's function, Geometry and Analysis, I, ALM 17, (2010), 285-302.

[9] F. Chung, P. Horn and A. Tsiatas. Distributing antidote using PageRank vectors. Internet Mathematics 6:2 (2009), 237-254.

[10] F. Chung and W. Zhao. A sharp PageRank algorithm with applications to edge ranking and graph sparsification. Preprint, http://www.math.ucsd.edu/ fan/wp/sharp.pdf.

[11] M.E. Dyer and A.M. Frieze. A simple heuristic for the $p$-centre problem. Operations Research Letters 3:6 (1985), 285-288.

[12] P. Eades and Q. Feng. Multilevel visualization of clustered graphs. Proceedings of the International Symposium on Graph Drawing (1996), 101-112.

[13] A.J. Enright, S. Van Dongen and C.A. Ouzounis. An efficient algorithm for large-scale detection of protein families. Nucleic Acids Research 30:7 (2002), $1575-1584$.

[14] M. Faloutsos, P. Faloutsos and C. Faloutsos. On power-law relationships of the Internet topology. Proceedings of the Conference on Applications, Technologies, Architectures, and Protocols for Computer Communication (SIGCOMM 1999), 251-262.

[15] S. Fortune. A sweepline algorithm for Voronoi diagrams. Proceedings of the Second Annual Symposium on Computational Geometry (1986), 313-322.

[16] E. Gansner and C. North. An open graph visualization system and its applications to software engineering. Software - Practice and Experience 30:11 (2000), 1203-1233.

[17] M. Girvan and M.E.J. Newman. Community structure in social and biological networks. Proceedings of the National Academy of Sciences 99:12, (2002), 7821-7826.

[18] V. Guruswami, D. Micciancio and O. Regev. The complexity of the covering radius problem on lattices and codes. Computational Complexity 14:2, (2005), 90-120. 
[19] D. Harel and Y. Koren. Graph drawing by high-dimensional embedding. Proceedings of the 10th International Symposium on Graph Drawing (2002), 207-219.

[20] G. Jeh and J. Widom. Scaling personalized Web search. Proceedings of the 12th International Conference on World Wide Web (2003), 271-279.

[21] T. Kamada and S. Kawai. An algorithm for drawing general undirected graphs. Information Processing Letters 31:1 (1989), 7-15.

[22] S. Lloyd. Least square quantization in PCM. IEEE Transactions on Information Theory 28:2 (1982), 129-137.

[23] L. Lovász and M. Simonovits, Random walks in a convex body and an improved volume algorithm, Random Structures and Algorithms 4 (1993), $359-412$.

[24] D. Lusseau, K. Schneider, O.J. Boisseau, P. Haase, E. Slooten and S.M. Dawson. The bottlenose dolphin community of Doubtful Sound features a large proportion of long-lasting associations. Behavioral Ecology and Sociobiology 54:4 (2003), 396-405.

[25] J. MacQueen. Some methods for classification and analysis of multivariate observations. Proceedings of the 5th Berkeley Symposium on Mathematical Statistics and Probability (1967), 281-297.

[26] S. Mancoridis, B.S. Mitchell, Y. Chen and E.R. Gansner. Bunch: a clustering tool for the recovery and maintenance of software system structures. Proceedings of the IEEE International Conference on Software Maintenance (1999), 50-59.

[27] J. Moody. Peer influence groups: identifying dense clusters in large networks. Social Networks 23:4 (2001), 261-283.

[28] M.E.J. Newman and M. Girvan. Finding and evaluating community structure in networks. Physical Review E 69 (2004), 026113.

[29] A. Ng, M. Jordan and Y. Weiss. On spectral clustering: analysis and an algorithm. Advances in Neural Information Processing Systems 14:2 (2002), 849-856.

[30] A. Noack. Modularity clustering is force-directed layout. Physical Review E 79 (2009), 026102.

[31] G. Parker, G. Franck and C. Ware. Visualization of large nested graphs in 3D: navigation and interaction. Journal of Visual Languages and Computing 9:3 (1998), 299-317.

[32] M. Rudelson and R. Vershynin. Sampling from large matrices: An approach through geometric functional analysis. Journal of the ACM 54:4 (2007), Article 21. 
[33] S.E. Schaeffer. Graph clustering. Computer Science Review 1:1 (2007), 2764.

[34] J. Shi and J. Malik. Normalized cuts and image segmentation. IEEE Transactions on Pattern Analysis and Machine Intelligence 22:8 (2000), 888-905. 